Facile route of flexible wire grid polarizer fabrication by angled-evaporations of aluminum on two sidewalls of an imprinted nanograting

This article has been downloaded from IOPscience. Please scroll down to see the full text article.

2012 Nanotechnology 23344018

(http://iopscience.iop.org/0957-4484/23/34/344018)

View the table of contents for this issue, or go to the journal homepage for more

Download details:

IP Address: 141.211.173.82

The article was downloaded on $26 / 06 / 2013$ at $14: 42$

Please note that terms and conditions apply. 


\title{
Facile route of flexible wire grid polarizer fabrication by angled-evaporations of aluminum on two sidewalls of an imprinted nanograting
}

\author{
Young Jae Shin ${ }^{1}$, Carlos Pina-Hernandez ${ }^{1,4}$, Yi-Kuei Wu ${ }^{2}$, Jong G Ok ${ }^{3}$ \\ and L Jay Guo ${ }^{1,2,3}$ \\ ${ }^{1}$ Macromolecular Sciences and Engineering, University of Michigan, Ann Arbor, MI 48109, USA \\ ${ }^{2}$ Department of Electrical Engineering and Computer Science, University of Michigan, Ann Arbor, MI \\ 48109, USA \\ ${ }^{3}$ Department of Mechanical Engineering, University of Michigan, Ann Arbor, MI 48109, USA \\ E-mail: guo@umich.edu
}

Received 13 March 2012, in final form 28 June 2012

Published 10 August 2012

Online at stacks.iop.org/Nano/23/344018

\begin{abstract}
In this study, we report a new method to fabricate a wire grid polarizer (WGP) that greatly relaxes the requirement on patterning and etching, and can be easily applied to produce flexible WGPs. The technique is to pattern a high aspect ratio and narrow linewidth grating by nanoimprint lithography followed by two angled aluminum depositions in opposite directions to produce the narrow spacing between the aluminum lines required for a visible band WGP. Anisotropic reactive ion etching is used to remove the aluminum deposited at the top of the grating but leave the aluminum layer on the grating sidewalls, thereby forming a metal wire grid with much smaller spacings than a lithographically defined grating. As a result, the fabricated WGP showed good performance in a wide range of visible wavelength.
\end{abstract}

(Some figures may appear in colour only in the online journal)

\section{Introduction}

A polarizer is an essential optical device for many optical systems and networks such as polarization-based imaging systems, free-space optical switching networks, and fiberoptic networks. Conventional polarizers are bulky optics and may not be suitable for certain applications. A metal wire grid is a potential candidate for making a high-quality, integration-capable, thin-film-type polarizer [1-8]. Wire grid polarizers (WGPs) offer a large spectral working range, small feature size and good integrability. WGPs are important for various applications such as microscopy or imaging systems, and can also be used as polarized beam splitters. The use of a WGP to replace the traditional polarizing film in an

4 Present address: aBeam Technologies, 5286 Dunnigan Ct., Castro Valley, CA, 94546, USA.
LCD can improve the LCD brightness as little light is absorbed by a WGP and reflected light can be recycled. Generally, a WGP consists of a periodical arrangement of conductive (metallic) wires on a transparent substrate. Upon illumination, a wire grid polarizer transmits light with an electrical field vector perpendicular to the wires (transverse magnetic (TM) polarization) with high efficiency, and reflects the parallel counterpart (transverse electric (TE) polarization). In addition to the transmission of TM-polarized light, the extinction ratio, which is defined by the transmission ratio of TM- and TE-polarized light, is another characteristic optical property of a wire grid polarizer. The spectral working range and the optical properties of a wire grid polarizer (such as transmittance and extinction ratio) are determined by the grating material and the structural parameters of the metal grating such as the period, grating height, and linewidth. In the last two decades various research results on the fabrication 
of WGPs using NILs have been reported [1-8]. Ekinci et al demonstrated the realization of a new bilayer metal WGP [9]. They fabricated the bilayer metal WGP by evaporating an aluminum film onto a poly(methyl methacrylate) resist grating fabricated using interference lithography. Hsu et al described the fabrication process of a flexible nano-wired polarizer by contact-transferred and mask embedded lithography using a polyurethane acrylate mold [10]. To pattern the fine pitch required for WGP, nanoimprint lithography (NIL) has been used in recent years due to its potential for high-resolution and high-throughput nanoscale patterning [11-14]. Previously we reported the fabrication of bilayer metal WGPs on flexible plastic substrates by roll-to-roll UV nanoimprint lithography using an epoxysilicone resist [15] and metal evaporation technique [16]. The high-throughput UV-NIL process has the potential to enable large area metal WGP fabrication [16, 17].

Displays have progressed toward increasingly thinner, lighter weight, and more flexible devices over the last few years. This is especially true in the field of mobile devices. Polarizers made on polymer substrate could find applications in future flexible LCD displays. WGPs require the fabrication of subwavelength metal gratings, which presents a significant challenge for patterning and etching of dense structures, particularly for use in visible wavelength applications. This becomes even more challenging to process on a flexible substrate. It is known that a WGP should have a period lower than $150 \mathrm{~nm}$ in order to provide adequate bandwidth to cover the visible light range. In this study, a flexible WGP was fabricated on a poly(ethylene terephthalate) (PET) film by using a new method with nanoimprint lithography (NIL). Even though the imprinted high aspect ratio grating had a relatively large $220 \mathrm{~nm}$ period, the WGP obtained using the new method still showed very good performance in the wide visible band of the spectrum. This was made possible by using double angled aluminum deposition to cover the sidewalls of the imprinted polymer grating; therefore a much narrower spacing between the aluminum lines was obtained. Isotropic reactive ion etching was then used to selectively remove the aluminum deposited at the top of the grating, thereby forming a metal wire grid with a much smaller spacing than a lithographically defined grating. This new approach significantly relaxes the stringent requirement for the fine line patterning process used in traditional methods, and is especially desirable for future manufacture of flexible WGPs using a roll-to-roll nanoimprint process.

\section{Experimental details}

NIL was performed on a flexible PET film in order to fabricate a flexible WGP. A nanograting of $220 \mathrm{~nm}$ period, $70 \mathrm{~nm}$ linewidth, and $200 \mathrm{~nm}$ height was imprinted into epoxy-silsesquioxane material. As compared with the material reported in [18], the epoxy-silsesquioxane used in this work was synthesized by using a larger amount of fluorinated compound to achieve higher aspect ratio structures. Then, two angled aluminum depositions were performed from both sides of the grating walls at $40^{\circ}$ angle from a normal direction. Aluminum from the upper part of the pattern was removed by reactive ion etching. The obtained WGP had a unique structure in which there were aluminum coatings on both sides of the wall of the nanopattern, creating a flexible WGP on PET.

\subsection{Materials and synthesis of $S S Q$}

To synthesize the SSQ material, phenyltrimethoxysilane (PTMS), (3-glycidyloxypropyl)trimethoxysilane (GTMS), (tridecafluoro-1-octyl)triethoxysilane (FTES), $\mathrm{CsOH}$, and propylene glycol monomethyl ether acetate (PGMA) were purchased from Aldrich Chemical (St Louis, MO, USA). Photoacid generator (PAG) was purchased from Craig Adhesive and Coating Co. (Newark, NJ, USA) under the compound name of UV9390C. It contained about 50 wt $\%$ of bis(4-dodecylphenyl)iodonium hexafluoroantimonate as an active ingredient. Synthesis of poly(phenyl-co3-gylcidoxypropyl-co-perfluorooctyl)silsesquioxane (epoxySSQ) was carried out by a modified method that has been reported in [18]. PTMS, FTES, and GTMS were used to synthesize epoxy-SSQ. The mold release agent $1 \mathrm{H}, 1 \mathrm{H}, 2 \mathrm{H}, 2 \mathrm{H}$-perfluorodecyltrichlorosilane (FDTS) was purchased from Gelest Inc. (Morrisville, PA, USA). Silquest A-187 silane (GTMS as the main ingredient) was purchased from Crompton Co. (Lisle, IL, USA). PET film was obtained from 3M Co. (St Paul, MN, USA) with a thickness of $50 \mu \mathrm{m}$.

\subsection{Instruments}

A Nanonex 2000 imprinting tool (Monmouth Junction, NJ, USA) with vacuum capability and wavelength for UV curing at $365 \mathrm{~nm}$ or a light curing system (ELC-430) from Electro-Lite Corporation (Bethel, Ct, USA) was used for UV-NIL. Aluminum was deposited by electron-beam evaporation. Reactive ion etching (RIE) was conducted in an LAM 9400 tool (LAM Research Corporation, Fremont, CA, USA). Scanning electron microscopy (SEM) was performed by using a Hitachi SU8000 scanning electron microscope (Tokyo, Japan). Transmission of TM and TE was measured by using an HR4000CG spectrometer (Ocean Optics Inc., Dunedin, FL, USA) and a Nikon Eclipse TE300 microscope (Tokyo, Japan), with the assistance of a high-quality polarizer.

\subsection{Nanoimprint process}

The formulation of the nanoimprint resist was prepared by dissolving the epoxy-SSQ resin in PGMA, followed by the addition of PAG (1 wt $\%$ of the epoxy-SSQ resin). The original silicon oxide master molds were vapor coated with FDTS in order to achieve easy mold release after the NIL processes. The epoxy-SSQ resin solution was spun on a flexible PET film substrate. The substrates were previously surface treated with $\mathrm{O}_{2}$ plasma and then vapor coated with Silquest A-187 silane as an adhesion promoter to the SSQ resist material. The imprinting process was performed under UV light exposure for a few seconds at room temperature. A Nanonex 2000 imprinting tool with vacuum capability for UV curing at $365 \mathrm{~nm}$ or a light curing system (ELC-430) from Electro-Lite Corporation was used for imprinting and curing. The imprinting pressure was typically 40 psi. 


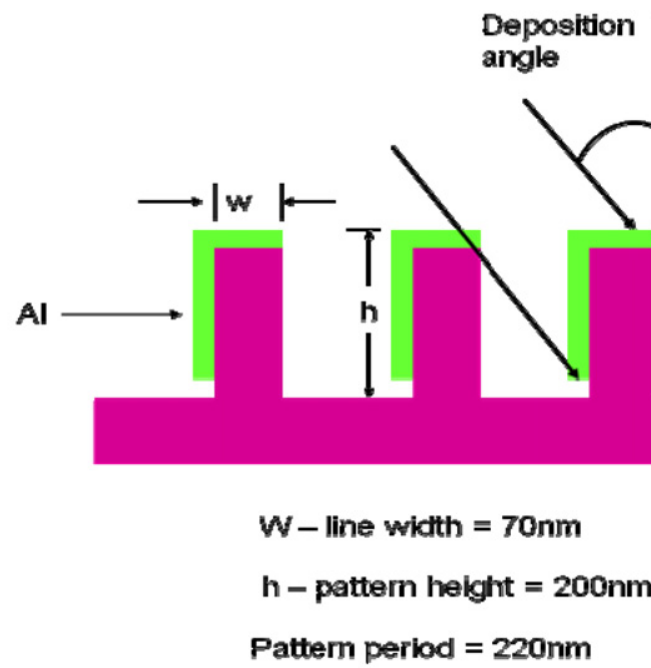

NIL Pattern

(a)
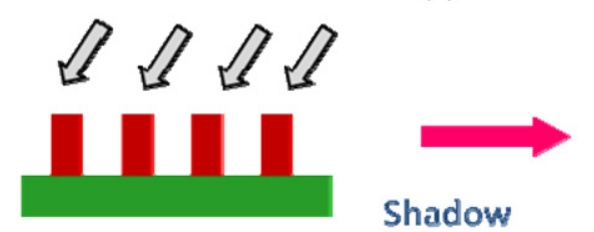

Shadow

evaporation
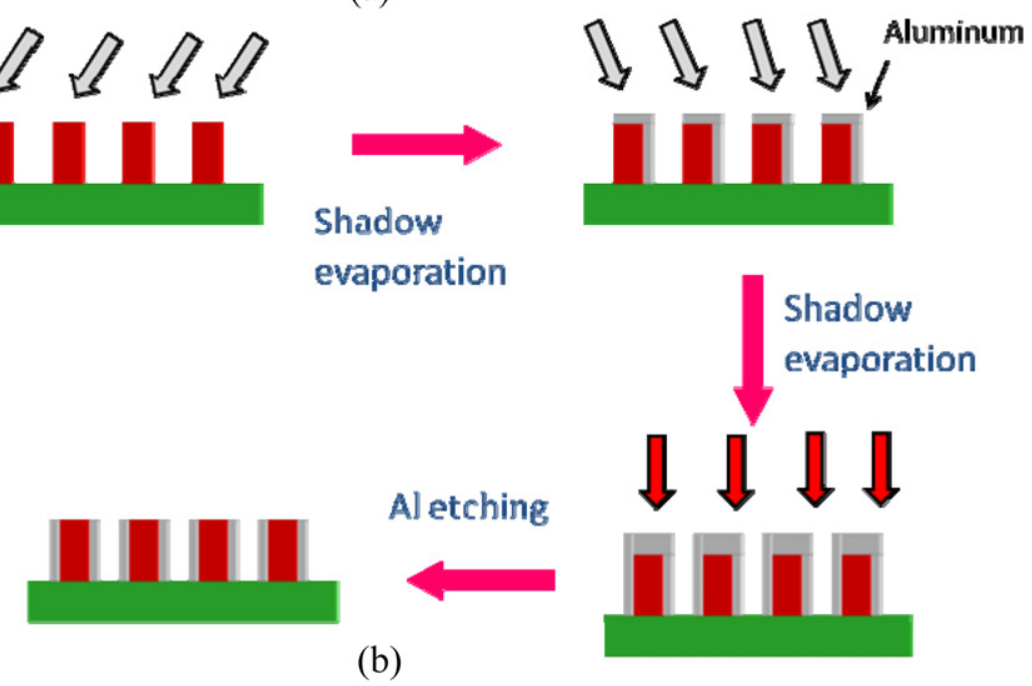

Figure 1. Tilted aluminum deposition on a nanopattern (a) and the total process involved in the fabrication of a WGP in this study (b).

\subsection{Aluminum deposition}

The rate of aluminum deposition in this study was $0.5 \mathrm{~nm} \mathrm{~s}^{-1}$. In order to deposit aluminum on the sidewalls of the imprinted nanopattern but not on the base of the trench, the deposition mount was tilted to $40^{\circ}$. The deposition process was repeated in the opposite direction to achieve aluminum deposition on both sidewalls of the SSQ nanogratings. This process is explained in figure 1(a).

\subsection{Plasma ion etching process}

For good polarizer performance, the aluminum layer deposited on top of the SSQ grating needs to be removed. We used an anisotropic plasma etching process to selectively remove the top aluminum while leaving the aluminum on the sidewalls of the grating relatively intact. Aluminum etching was performed in the LAM 9400 tool. The aluminum etching had to be conducted in two steps under $200 \mathrm{~W}$ transformer coupled plasma RF power. First, any residual aluminum oxide layer (thickness $\sim 1 \mathrm{~nm}$ ) was etched by using $\mathrm{BCl}_{3}$ plasma
(40 sccm) for $5 \mathrm{~s}$, followed by the aluminum layer etching by using $\mathrm{BCl}_{3}(20 \mathrm{sccm}) / \mathrm{Cl}_{2}(6 \mathrm{sccm})$ plasma.

The overall fabrication process of the WGP is illustrated in figure 1(b).

\section{Results and discussion}

\subsection{Design considerations for the WGP}

The purpose of this study was to fabricate a WGP which showed good performance across a wide wavelength range of visible light by using NIL. It is well known that typical WGPs must have a period lower than $150 \mathrm{~nm}$ in order to be used in the visible light range, as was achieved in previous WGPs fabricated using NIL. However it is challenging to fabricate molds with smaller periods and imprint with high yields. To relax the fabrication challenges, a method to create efficient WGPs by using molds with larger periods is desirable.

Our new method to fabricate WGPs used angled aluminum depositions on the nanoimprinted pattern from both sides at a $40^{\circ}$ angle from the normal direction. As shown 

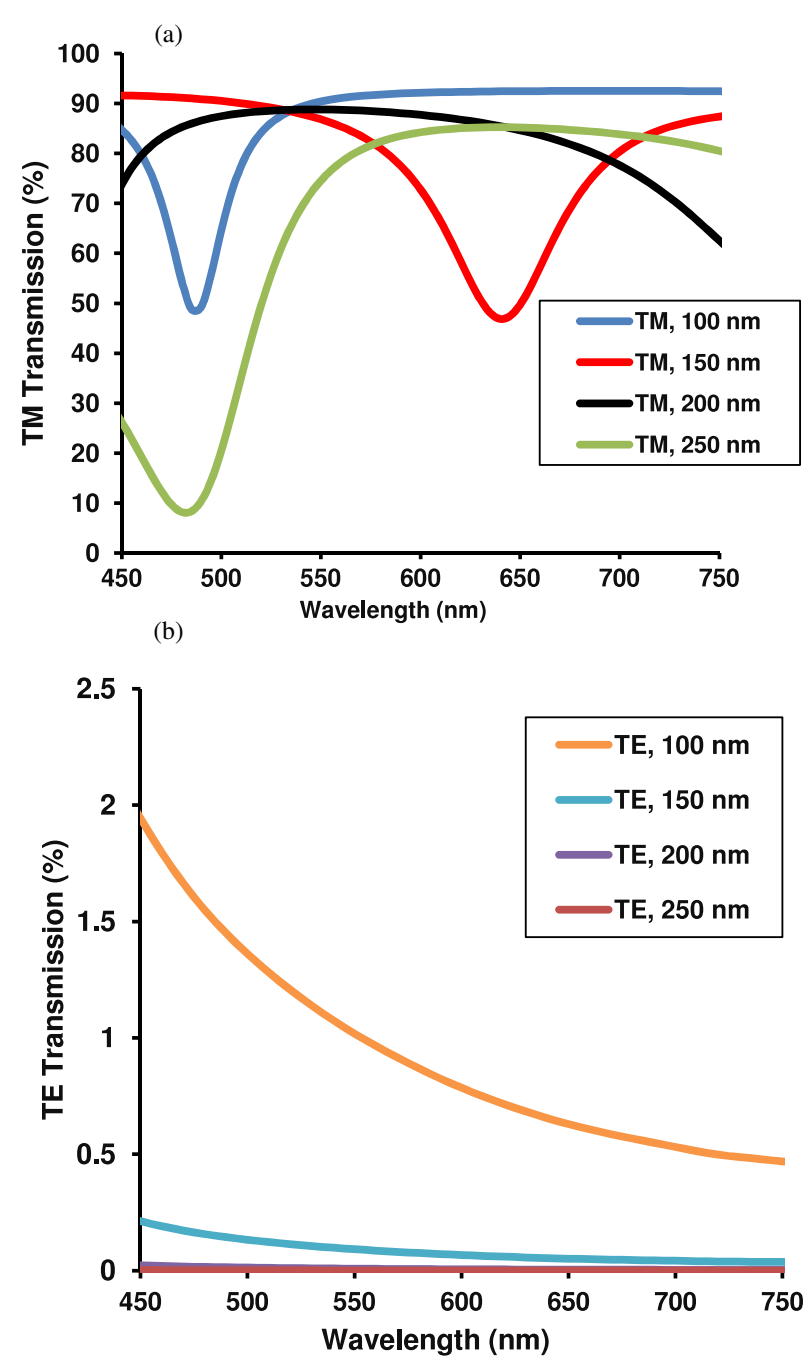

Figure 2. Simulation results of TM (a) and TE (b) for WGPs that contain different heights of the nanopattern $(220 \mathrm{~nm}$ period, $70 \mathrm{~nm}$ linewidth, $30 \mathrm{~nm}$ thickness of aluminum).

in figure 1(b), aluminum from the upper part of the pattern was removed by reactive ion etching. The final structure of the WGP had aluminum coating on both sides of the nanoimprinted grating walls. A nanoimprinted pattern with a $220 \mathrm{~nm}$ period and a $70 \mathrm{~nm}$ linewidth was prepared by using NIL in this study to leave room for the aluminum coating.

We carried out a simulation of the performance of the WGP by using the COMSOL program to design the structure and optimize its polarization performance. The performance of the WGP was characterized by the TM transmittance and extinction ratio, defined as the ratio of transmittance of the TM-polarized light to that of the TE-polarized light. Fixing the period at $220 \mathrm{~nm}$, the linewidth at $70 \mathrm{~nm}$, and the sidewall Al coating at $30 \mathrm{~nm}, \mathrm{TM} / \mathrm{TE}$ transmission simulation results for heights varying from 100 to $250 \mathrm{~nm}$ are shown in figure 2. The dips in the transmission curve are the result of Fabry-Perot resonances formed within the metallic nanoslits [19]. The WGP with a $200 \mathrm{~nm}$ height showed relatively flat TM transmission over the visible region as compared with the others.

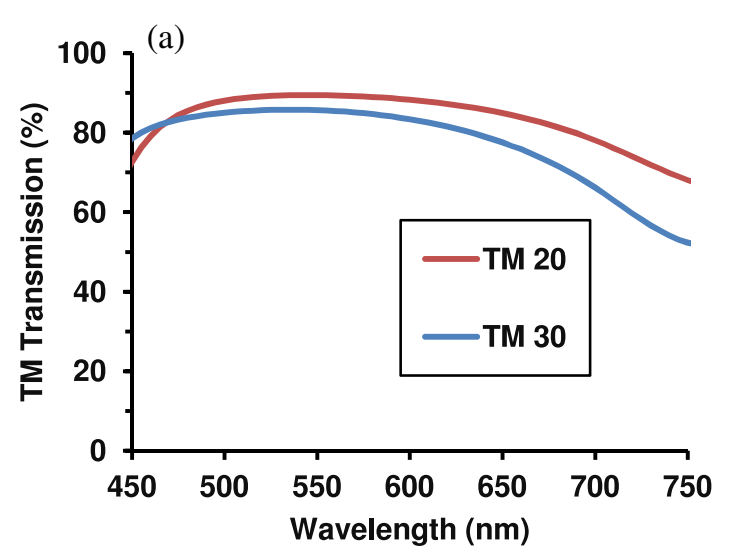

(b)

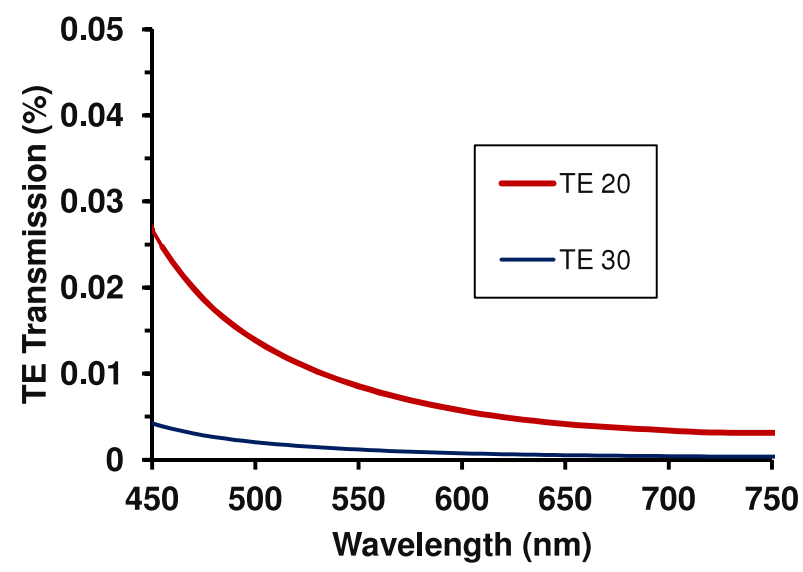

Figure 3. Simulation results of TM (a) and TE (b) for WGPs that contain epoxy-SSQ nanopatterns with 20 or $30 \mathrm{~nm}$ aluminum deposition on both the sidewalls $(220 \mathrm{~nm}$ period, $70 \mathrm{~nm}$ linewidth, $200 \mathrm{~nm}$ grating height).

We also simulated the performance of the WGP by changing the thickness of the aluminum deposition from 15 to $35 \mathrm{~nm}$. The simulation results of TM values and TE values at the different wavelengths for the WGPs are shown in table 1. Simulation results in the whole wavelength range of visible light for WGPs with 30 or $20 \mathrm{~nm}$ aluminum deposition on the sidewalls are shown in figure 3.

\subsection{Synthesis of epoxy-SSQ and NIL}

Epoxy-SSQ was synthesized from PTMS, FTES, and GTMS. The PTMS component helps to increase the hardness of the synthesized SSQ polymer and the FTES helps to improve the releasing property of the imprinted SSQ patterns from the mold. GTMS contains the epoxy functional group which can polymerize during NIL via cationic polymerization, and make it immune to the oxygen environment. Epoxy-SSQ was synthesized by using a method that was modified from a previously reported method [18] used by our group. The epoxy functional group shows good mechanical properties after the completion of curing, and simultaneously shows good adhesion. This property plays an important role in an NIL process to help the adhesion of the imprinted material with the substrate. Better mold releasing is also very important 

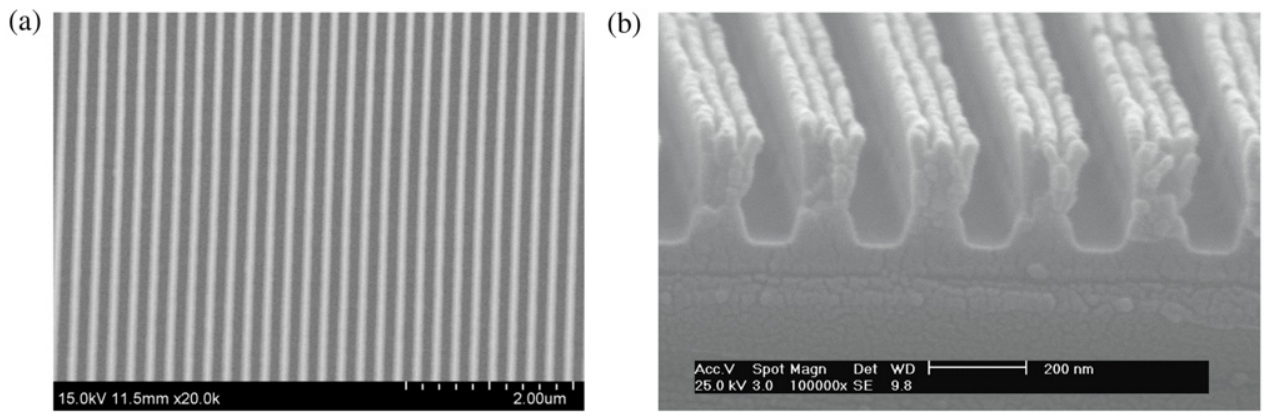

Figure 4. SEM photograph of the SSQ nanopattern on a flexible PET film (a) and the cross section of a nanopattern after aluminum deposition and ion etching (b).

Table 1. Simulation results of transmission (\%) of TM/TE-polarized light at the different wavelengths for WGPs with different thicknesses of aluminum deposition.

\begin{tabular}{lllll}
\hline Thickness $(\mathrm{nm})$ & $450 \mathrm{~nm}$ & $550 \mathrm{~nm}$ & $650 \mathrm{~nm}$ & $750 \mathrm{~nm}$ \\
\hline 15 & $47.4 / 0.789$ & $91.8 / 0.268$ & $91.9 / 0.131$ & $83.6 / 0.0875$ \\
20 & $72.6 / 0.0267$ & $89.5 / 0.00853$ & $84.9 / 0.00414$ & $68.1 / 0.00311$ \\
25 & $69.9 / 0.0678$ & $90.0 / 0.244$ & $87.2 / 0.0123$ & $69.5 / 0.00835$ \\
30 & $78.6 / 0.00423$ & $85.7 / 0.00118$ & $79.5 / 0.000507$ & $62.3 / 0.000354$ \\
35 & $74.3 / 0.00869$ & $87.1 / 0.00299$ & $81.7 / 0.00149$ & $56.4 / 0.00103$ \\
\hline
\end{tabular}

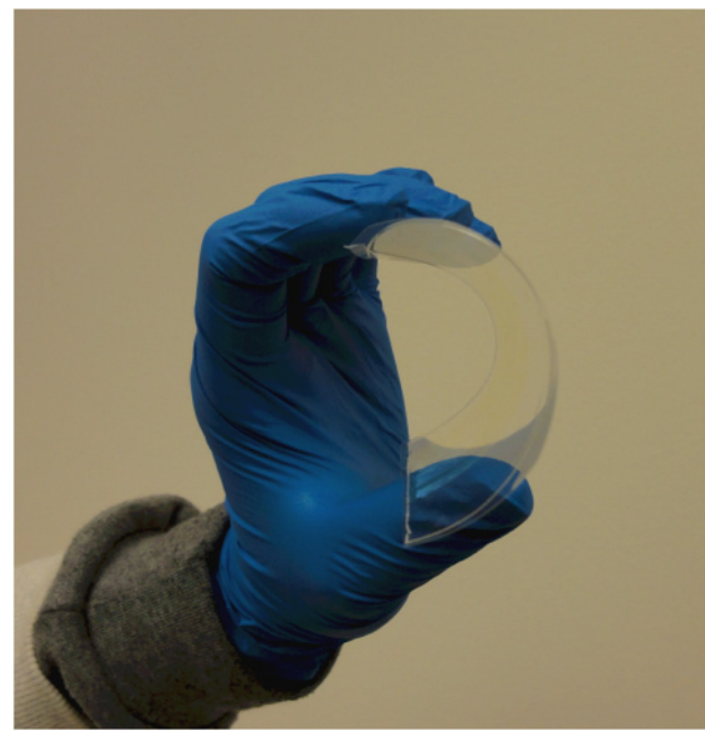

Figure 5. Flexibility of a PET film containing the WGP structure.

to ensure defect-free patterning over a large area. This was achieved by increasing the amount of FTES in the resist formulation. The SEM of the formed SSQ nanograting pattern is shown in figure 4(a), showing a well defined high aspect ratio and uniform nanopattern. Most notably a very regular and uniform pattern without any defects was obtained in a large area, due to the increase in the amount of FTES in the resist formulation for ease of demolding.

\subsection{Aluminum deposition and etching}

In figure 1(b), the structure of the obtained WGP was shown with the aluminum coating on both sides of the $70 \mathrm{~nm}$ nanopattern line. The SEM photograph of the fabricated WGP after aluminum deposition and etching is shown in

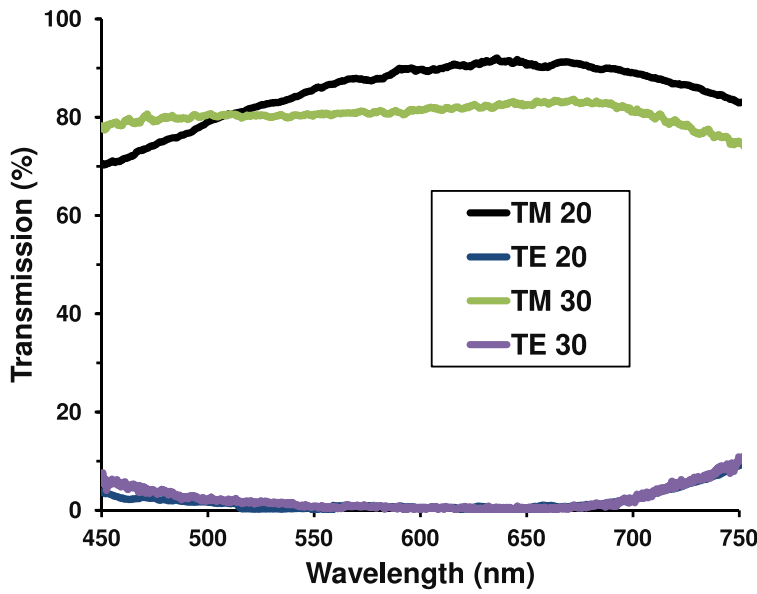

Figure 6. The TE and TM curves of a nanopatterned epoxy-SSQ WGP with 20 or $30 \mathrm{~nm}$ aluminum deposition on both the sidewalls (220 nm period, $70 \mathrm{~nm}$ linewidth, $200 \mathrm{~nm}$ grating height).

figure 4(b), where the aluminum layers on both sidewalls of the nanopatterns can be clearly seen. Note that this structure was damaged slightly by the reactive ion etching process.

As the WGP structure was fabricated on a PET film, the final WGP sheet was very flexible as shown in figure 5.

\subsection{Measurement of TM and TE transmission and extinction ratio}

The optical properties of the obtained WGP were estimated by measuring the transmittance of both the TM and the TE polarized light. The results of the measurements of TM and TE transmission for the fabricated WGPs are shown in table 2. The results in the whole wavelength range of visible light for WGPs with 30 or $20 \mathrm{~nm}$ aluminum deposition on the sidewalls ae shown in figure 6 . 
Table 2. Measured optical transmission (\%) of TM/TE-polarized light through nanopatterned epoxy-SSQ/aluminum WGPs at different wavelengths for WGPs with different thicknesses of aluminum deposition on the SSQ grating sidewalls.

\begin{tabular}{lllll}
\hline Thickness $(\mathrm{nm})$ & $450 \mathrm{~nm}$ & $550 \mathrm{~nm}$ & $650 \mathrm{~nm}$ & $750 \mathrm{~nm}$ \\
\hline 15 & $50.6 / 6.53$ & $90.3 / 0.670$ & $89.7 / 0.179$ & $82.1 / 9.43$ \\
20 & $70.5 / 4.65$ & $85.8 / 0.082$ & $90.6 / 0.031$ & $82.9 / 9.09$ \\
25 & $71.1 / 5.09$ & $89.5 / 0.097$ & $81.7 / 0.104$ & $75.9 / 11.3$ \\
30 & $77.7 / 5.02$ & $80.3 / 0.100$ & $82.4 / 0.039$ & $74.7 / 10.4$ \\
35 & $70.8 / 5.12$ & $78.9 / 0.377$ & $80.1 / 0.235$ & $68.2 / 11.2$ \\
\hline
\end{tabular}

The case of the WGP with $30 \mathrm{~nm}$ aluminum sidewall coating is shown in figure $6.75-85 \%$ of the TM polarizedlight was transmitted in the wide wavelength range of visible light. On the other hand, below 5\% of the TE polarized-light was transmitted in the same wavelength range. The case of the WGP with $20 \mathrm{~nm}$ aluminum coating is also shown in figure 6 . $70-90 \%$ of the TM polarized-light was transmitted in the wide wavelength range of visible light.

The extinction ratio (ratio of TM to TE) for the $30 \mathrm{~nm}$ coated WGP showed values of 803 at $550 \mathrm{~nm}$, and 2117 at $650 \mathrm{~nm}$, while the $20 \mathrm{~nm}$ coated WGP showed ratios of 1046 at $550 \mathrm{~nm}$, and 2900 at $650 \mathrm{~nm}$. The TM transmission in figure 6 showed a flatter curve in the wide wavelength range of visible light.

\section{Conclusion}

Nanopatterns of $220 \mathrm{~nm}$ period, $70 \mathrm{~nm}$ linewidth, and $200 \mathrm{~nm}$ height were formed using synthesized epoxy-SSQ on a flexible PET film. Angled aluminum depositions were performed at a $40^{\circ}$ angle from the perpendicular plane and aluminum was removed from the top of the pattern by reactive ion etching. The obtained WGP had a unique structure in which the aluminum coating was on both sides of the walls of the nanopattern. Despite using a larger period than previously reported WGPs, high extinction ratios were achieved over the visible spectrum with a simple fabrication process. If these results can be migrated to roll-to-roll processing methods [16, 17], it will be possible to easily manufacture efficient WGPs over large areas.

\section{Acknowledgments}

This work was supported in part by NSF and DARPA through subcontract from Innovega Inc. Nanofabrication was performed at the Lurie Nanofabrication Facility (LNF), and electron microscopy analysis was performed at the Electron Microbeam Analysis Laboratory (EMAL) at the University of Michigan.

\section{References}

[1] Golden J H, DiSalvo F J, Silcox J, Thomas M and Elman J 1996 Subnanometer-diameter wires isolated in a polymer matrix by fast polymerization Science $\mathbf{2 7 3} 782$

[2] Liu X, Deng X, Sciortino J P, Buonanno M, Walters F, Varghese R, Bacon J, Chen L, O'Brien N and Wang J J 2006 Large area, 38 nm half-pitch grating fabrication by using atomic spacer lithography from aluminum wire grids Nano Lett. 62723

[3] Pang Y T, Meng G W, Zhang L D, Qin Y, Gao X Y, Zhao A W and Fang Q 2002 Arrays of ordered Pb nanowires and their optical properties for laminated polarizers Adv. Funct. Mater. 12719

[4] Ahn S W, Lee K D, Kim J S and Kim S H 2005 Fabrication of a $50 \mathrm{~nm}$ half-pitch wire grid polarizer using nanoimprint lithography Nanotechnology 161874

[5] Wang J J, Chen L, Liu X M, Sciortino P, Liu F, Walters F and Deng X G $200630 \mathrm{~nm}$ wide aluminum nanowire grid for ultrahigh contrast and transmittance polarizers made by UV-nanoimprint lithography Appl. Phys. Lett. 89141105

[6] Wang J J, Walters F, Liu X M, Sciortino P and Deng X G 2007 High-performance, large area, deep ultraviolet to infrared polarizers based on $40 \mathrm{~nm}$ line/78 $\mathrm{nm}$ space nanowire grids Appl. Phys. Lett. 90061104

[7] Pais A, Banerjee A, Klotzkin D and Papautsky I 2008 High-sensitivity, disposable lab-on-a-chip with thin-film organic electronics for fluorescence detection Lab Chip 8794

[8] Wang J J, Zhang W, Deng X, Deng J, Liu F, Sciortino P and Chen L 1995 High-performance nanowire-grid polarizers Opt. Lett. 30195

[9] Ekinci Y, Solak H H, David C and Sigg H 2006 Bilayer Al wire-grids as broad band and high-performance polarizers Opt. Express 142323

[10] Hsu C C and Lee Y C 2010 Fabrication of flexible nano-wired polarizer by contact-transferred and mask embedded lithography and polyurethaneacrylate mold Nano/Micro Engineered and Molecular Systems (NEMS) 5th IEEE Int. Conf. (Jan 2010) pp 893-7

[11] Chou S Y, Krauss P R and Renstrom P J 1995 Imprint of sub-25 nm vias and trenches in polymers Appl. Phys. Lett. 673114

[12] Chou S Y, Krauss P R and Renstrom P J 1996 Imprint lithography with $25 \mathrm{~nm}$ resolution Science $\mathbf{2 7 2} 85$

[13] Guo L J 2007 Nanoimprint lithography: methods and material requirements Adv. Mater. 19495

[14] Choi P, Fu P F and Guo L J 2007 Siloxane copolymers for nanoimprint lithography Adv. Funct. Mater. 1765

[15] Cheng X, Guo L J and Fu P F 2005 Room temperature and low pressure nanoimprinting based on cationic photopolymerization of novel epoxysilicone monomers Adv. Mater. 171419

[16] Ahn S H and Guo L J 2008 High-speed roll-to-roll nano imprint lithography on flexible plastic substrates $A d v$. Mater. 202044

[17] Ahn S H and Guo L J 2009 Large-area roll-to-roll and roll-to-plate nanoimprint lithography and analytical models for predicting residual layer thickness ACS Nano 32304

[18] Pina-Hernandez C, Guo L J and Fu P F 2010 High-resolution functional epoxysilsesquioxane-based patterning layers for large-area nanoimprinting ACS Nano 44776

[19] Shi H and Guo L J 2012 An experimental study of light funneling effect into nano-grooves on gold film Phys. Rev. B submitted 